\title{
Disorders of Consciousness due to Traumatic Brain Injury: Functional Status Ten Years Post-Injury
}

\author{
Flora M. Hammond, ${ }^{1}$ Joseph T. Giacino, ${ }^{2}$ Risa Nakase Richardson, ${ }^{3,4}$ Mark Sherer, ${ }^{5}$ Ross D. Zafonte, ${ }^{6}$ \\ John Whyte, ${ }^{7}$ David B. Arciniegas, ${ }^{8}$ and Xinyu Tang ${ }^{9}$
}

\begin{abstract}
Few studies have assessed the long-term functional outcomes of patients with a disorder of consciousness due to traumatic brain injury (TBI). This study examined functional status during the first 10 years after TBI among a cohort with disorders of consciousness (i.e., coma, vegetative state, minimally conscious state). The study sample included 110 individuals with TBI who were unable to follow commands prior to inpatient rehabilitation and for whom follow-up data were available at 1,2,5, and 10 years post-injury. The sample was subdivided into those who demonstrated command-following early (before 28 days post-injury) versus late ( $\geq 28$ days post-injury or never). Functional Independence Measure (FIM) at 1, 2, 5, and 10 years following TBI was used to measure functional outcomes. Measureable functional recovery occurred throughout the 10-year period, with more than two thirds of the sample achieving independence in mobility and self-care, and about one quarter achieving independent cognitive function by 10 years. Following commands prior to 28 days was associated with greater functional independence at all outcome time-points. Multi-trajectory modeling of recovery of three FIM subscales (self-care, mobility, cognition) revealed four distinct prognostic groups with different temporal patterns of change on these subscales. More than half the sample achieved near-maximal recovery by 1 year post-injury, while the later command-following subgroups recovered over longer periods of time. Significant late functional decline was not observed in this cohort. Among a cohort of patients unable to follow commands at the time of inpatient rehabilitation, a substantial proportion achieved functional independence in self-care, mobility, and cognition. The proportion of participants achieving functional independence increased between 5 and 10 years post-injury. These findings suggest that individuals with disorders of consciousness may benefit from ongoing functional monitoring and updated care plans for at least the first decade after TBI.
\end{abstract}

Keywords: brain injuries; cognition; consciousness disorders; minimally conscious state; prognosis; rehabilitation outcome; vegetative state

\section{Introduction}

$\mathbf{F}$ EW STUDIES HAVE ASSESSED the long-term functional outcomes of patients with disorders of consciousness (DOC) due to traumatic brain injury (TBI). In addition, many utilized broad, non-specific outcome measures with variable samples at follow-up intervals. There is consequently a lack of data to guide families, providers, and payers in trying to plan for future needs, compare outcomes, and assess quality of interventions. Given the lack of knowledge regarding meaningful long-term outcomes, it is not

\footnotetext{
${ }^{1}$ Department of Physical Medicine and Rehabilitation, Indiana University School of Medicine, Indianapolis, Indiana.

${ }^{2}$ Department of Physical Medicine and Rehabilitation, Harvard Medical School, Boston, Massachusetts.

${ }^{3}$ Health Services Research and Development Service, Center of Innovation for Disability and Rehabilitation Research, and Defense and Veterans Brain Injury Center, James A. Haley Veterans Hospital, ${ }_{5}^{4}$ College of Medicine, University of South Florida, Tampa, Florida.

${ }^{5}$ TIRR Memorial Hermann, Houston, Texas.

${ }^{6}$ Spaulding Rehabilitation Hospital, Massachusetts General Hospital, Brigham and Women's Hospital, Department of Physical Medicine and Rehabilitation, Harvard Medical School, Boston, Massachusetts.

${ }^{7}$ Moss Rehabilitation Research Institute, Elkins Park, Pennsylvania.

${ }^{8}$ Departments of Neurology and Psychiatry, Center for Mental Health, Marcus Institute for Brain Health, University of Colorado School of Medicine, Gunnison, Colorado.

${ }^{9}$ Department of Pediatrics, University of Arkansas for Medical Sciences, Little Rock, Arkansas.

(c) Flora M. Hammond et al., 2018; Published by Mary Ann Liebert, Inc. This Open Access article is distributed under the terms of the Creative Commons Attribution Noncommercial License (http://creativecommons.org/licenses/by-nc/4.0/) which permits any noncommercial use, distribution, and reproduction in any medium, provided the original authors and the source are credited.
} 
surprising that healthcare providers tend to provide pessimistic prognostic information. ${ }^{1,2}$ It is possible such low prognostic expectations influence care and outcomes. In fact, in the United States, individuals on mechanical ventilation with a Glasgow Coma Scale (GCS) score of 5 or less are referred for organ procurement. ${ }^{3}$ Many providers and payers deem patients with DOC who lack command-following as inappropriate for acute inpatient rehabilitation. $^{4,5}$ Thus, there is great need for data on specific long-term functional outcomes to inform post-acute treatment recommendations.

In contrast to these poor outcome expectations, recent literature has supported a more favorable outcome trajectory for those admitted to inpatient rehabilitation. Several studies have shown recovery of consciousness and functional independence during the first year following TBI for many individuals with DOC. ${ }^{6}$ Greater recovery is generally observed for those who are in the minimally conscious state as compared with the vegetative state at rehabilitation admission. ${ }^{6-12}$

Studies of the Traumatic Brain Injury Model Systems (TBIMS) cohort have shown that of 396 TBIMS participants who were admitted to inpatient rehabilitation unable to follow commands, 268 (68\%) regained command-following and, of these, 91 (23\%) emerged from post-traumatic amnesia prior to rehabilitation discharge. ${ }^{10}$ Of those who had failed to recover command-following by rehabilitation discharge, the majority of those with follow-up were able to follow commands by post-injury Years $1(59 \% ; n=46 /$ $78), 2(66 \% ; n=31 / 47)$, and $5(74 \% ; n=25 / 34)$. Of $337,20 \%$ were living without in-house supervision, and $19 \%$ were competitively employable. This study found significant improvements in FIM Motor and Cognition subscale scores from rehabilitation discharge to Year 1, Year 1 to 2, and Year 2 to $5(N=108)$. To better understand these improvements, Whyte and colleagues ${ }^{13}$ studied the changes in individual FIM items over the first 5 years post-injury. Among those who recovered command-following during inpatient rehabilitation, 56 to $85 \%$ (depending on the FIM item) were functionally independent by 5 years. The proportion with independent function across FIM items increased from each assessment interval to the next. For those without command-following by rehabilitation discharge, 19 to $36 \%$ were functioning independently by 5 years. In summary, those without return of commandfollowing during inpatient rehabilitation showed less functional progress than with command-following recovery during rehabilitation, though clinically meaningful change after rehabilitation discharge was observed.

These studies highlight important prognostic information in the first 5 years following TBI resulting in DOC. This issue has taken on great significance in the context of recent conceptualization of TBI as a chronic disease ${ }^{14}$ and preliminary evidence that some persons with TBI show functional decline by 5 years post-injury. ${ }^{15}$ However, outcomes of well-characterized samples with traumatic DOC beyond 5 years post-injury have not been described in the literature. The present study uses the same TBIMS cohort as Nakase-Richardson and colleagues ${ }^{10}$ and Whyte and colleagues ${ }^{13}$ to assess the level of independence achieved over the first 10 years post-injury. Additionally, this study examined whether there were distinct subgroups of DOC patients with characteristic patterns of recovery or decline. As in the study by Whyte and colleagues, ${ }^{13}$ the sample was subdivided into those who regained commandfollowing earlier versus later. However, many other studies define prolonged DOC as lasting more than 28 days. Thus, to study patterns of functional recovery over the first 10 years post-injury, the cohort was by subdivided by timing of following command post- injury: those who first followed commands prior to 28 days postinjury and those that did not. We hypothesized that function would change from 5 to 10 years post-injury with distinct recovery groups identified.

\section{Methods}

\section{Study participants}

Participants are consecutive inpatient rehabilitation admissions who were prospectively enrolled in the National Institute on Disability, Independent Living, and Rehabilitation Research (NIDILRR) funded TBIMS multi-site longitudinal registry with no evidence of command-following prior to rehabilitation admission (verified across two TBIMS variables as defined below) and completed 1-, 2-, 5-, and 10-year follow-ups. TBIMS database participants for this study were discharged from acute inpatient rehabilitation from January 1, 1989, through December 31, 2015. TBI is defined by at least one of the following: GCS score $<13$ on emergency department admission, loss of consciousness $>30 \mathrm{~min}$, post-traumatic amnesia $>24 \mathrm{~h}$, or trauma-related intracranial abnormality on neuroimaging. Additional TBIMS inclusion criteria are: age 16 years or older at the time of injury, received medical care in a TBIMS-affiliated trauma center within $72 \mathrm{~h}$ of injury, transferred directly to a TBIMS affiliated inpatient TBI rehabilitation program, and informed consent provided by legal proxy or participant. Excluded from the sample were participants who had incomplete follow-up at $1,2,5$, or 10 years post-injury, were missing functional outcome (FIM) data, or were dead. Those with FIM data at all four intervals but missing individual FIM items were included. All TBIMS sites have approval by their local institutional review board.

\section{Measures}

Disorders of consciousness. Participants were coded as having DOC based on command-following status across two TBIMS variables at the time of rehabilitation admission. ${ }^{10,13}$ The first variable was the date a participant exhibited commandfollowing on two consecutive examinations within a 24-h period following TBI. Acute rehabilitation medical records including nursing flow sheets and progress notes were exhaustively reviewed to determine if command-following was demonstrated prior to rehabilitation hospital admission. Such documentation might include mention of command-following or a GCS Motor score of 6 (indicating command-following). The second is the GCS Motor score coded as part of the Disability Rating Scale collected at rehabilitation admission. Participants were coded as having DOC if both variables indicated that no command-following ability prior to or at the time of inpatient rehabilitation admission.

FIM. The FIM ${ }^{16}$ measures functional independence or burden of care with 18 items that assess Self-care (six items), Mobility (seven items), and Cognition (five items). The bath to shower transfer item was not used in the present study given other transfer types being represented. Items are scored 1 through 7 with a score of 1 representing complete dependence and a score of 7 indicating complete independence.

\section{Procedures}

Trained TBIMS research assistants collected information regarding injury severity (GCS, time to follow commands [TFC]), and medical course from hospital and emergency medical service records consistent with the protocol for the TBIMS database. TFC is the interval in days from the date of injury until the first of two consecutive reports of command-following within a 24-h period. Demographic information such as date of birth, education, and pre- 
morbid functioning were collected in interview with the subjects or family/significant others. The Disability Rating Scale (including the GCS motor score) was administered at the time of rehabilitation admission. Follow up data were collected at 1, 2, 5, and 10 years post-injury using a standardized telephone follow-up assessment protocol. ${ }^{17}$ If the patient was not able to provide accurate information, data were collected from family members or care providers who were familiar with the participant.

\section{Statistical analysis}

Data were analyzed using statistical software $\mathrm{R}$ ( $\mathrm{R}$ Foundation for Statistical Computing, Vienna, Austria) for data manipulation and descriptive statistics, and SAS (SAS Institute Inc., Cary, NC; https://www.andrew.cmu.edu/user/bjones/download.htm) macro for group-based multi-trajectory models. ${ }^{18}$ Descriptive statistics were expressed as quartiles (1st/median/3rd) for continuous variables and count (percent) for categorical variables. The proportion of the sample that was Independent $(F I M=6-7)$, required Some Assistance (FIM =2-5), or was Totally Dependent (FIM= 1), was determined for each FIM item. For ambulation, independence level was determined using primary mobility mode (walking vs. wheelchair). The total Self-care, Mobility and Cognition indices were calculated as the sums of the respective item scores. To calculate summary scores for Self-care, Mobility, and Cognition, mean scores $>5.5$ indicated Independent, mean scores $>1.5$ to 5.5 indicated Some Assistance needed, and mean scores from 1 to 1.5 indicated Total Dependence. In cases of missing FIM scores, the data were omitted for that individual.

Group-based multi-trajectory modeling was carried out to identify latent clusters of individuals who followed similar trajectories of function over time with regard to Self-care, Mobility, and Cognition. Censored normal distributions were used for modeling the scores as both Self-care and Mobility scores ranged from 6 to 42, and the cognition score ranged from 5 to 35 . Because it is challenging to identify trajectory patterns over time on multiple scores, we fitted trajectory models for each FIM subscale separately to clarify the types of distinctive trajectories represented in our study sample. Trajectories were assumed to follow a quadratic function of follow-up years except for participants who were at floor or ceiling (i.e., Total Dependence or Independent) at 1-year follow-up and stayed the same across years. Models for up to five groups were explored. While the five-group multi-trajectory model had a slightly better Bayesian Information Criterion (BIC) score than the four-group model, the fivegroup model did not include a group that was substantively distinct from those in the four-group model. The performance of the fourgroup multi-trajectory model was assessed. The average posterior probability for those assigned to a group based on the maximum posterior probability rule was $99.2 \%$, which is far beyond the $70 \%$ threshold of acceptability. The proportion assigned to each group closely matched the estimated probability of group assignment.

\section{Results}

\section{Patient characteristics}

Figure 1 summarizes the number excluded from analyses by reason and the number lost-to-follow up with a final sample of 110 who met study criteria. Among the TBIMS national database participants, 664 met the case definition for a disorder of consciousness, as defined above. Of those, 530 were excluded due to: missing at least one follow up at Year 1, 2, 5, or $10(n=325)$, dead at Year 1, 2,5 , or $10(n=91)$, center no longer funded to follow the participant $(n=23)$, or missing 10-year FIM data $(n=4)$. Overall sample and subgroup characteristics (based on recovery trajectory group) are summarized in Table 1. Comparison of the sample that had consistent follow-up 10 years to those excluded due to inconsistent follow-up revealed similarities, except the study sample had more TBI due to motor vehicle crash and more chemically paralyzed.



FIG. 1. Participant flow diagram. 


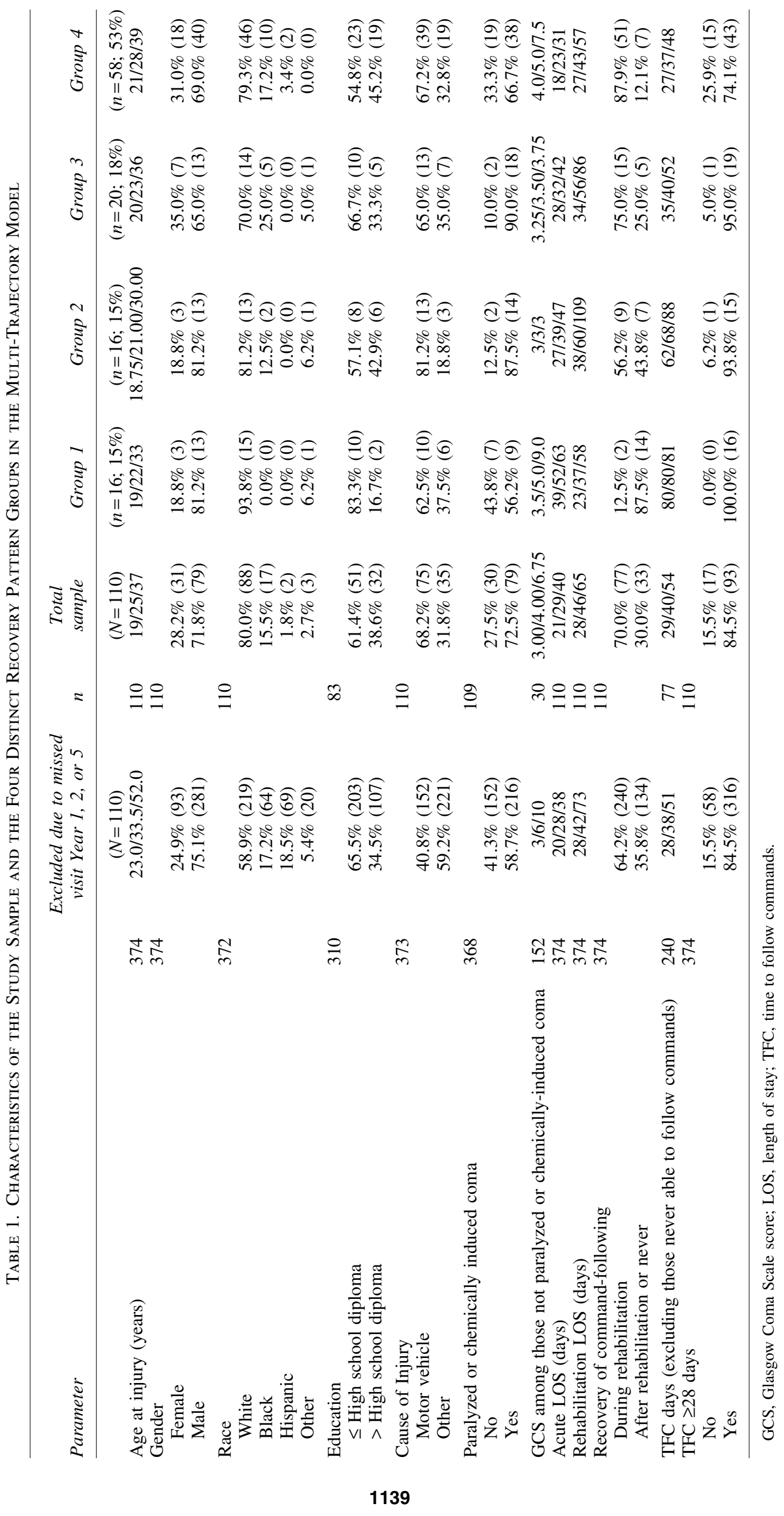


Proportion of individuals who were functionally independent

Table 2 shows the proportion and number of individuals who were Independent, required Some Assistance, and Total Dependence at each of the summary areas of function and each FIM item at the follow-up periods subdivided into those with TFC before versus after 28 days post-injury. In general, both subsamples demonstrated functional gains across the three FIM domains from Year 1 to 2, Year 2 to 5, and Year 5 to 10. Although the percentage of individuals improving to full independence between Years 5 and 10 was relatively small, increased proportions were noted across all but three FIM items. For both subsamples, all three FIM domains showed increases in the proportion of independent individuals between 5 and 10 years and decreases in the proportion of Totally Dependent individuals. Among the group who regained command-following before 28 days, $88-100 \%$ were independent across the three FIM subscores by 10 years, compared with 50 $75 \%$ for those who did not. Supplementary Figures 1 through 3 summarize the proportion independent at each epoch for the entire sample and by TFC timing subsample using a more visually friendly format (see online supplementary material at http:// www.liebertpub.com).

For direct comparison to Whyte and colleagues ${ }^{13}$ Supplementary Table 1, Supplementary Figure 4, and Supplementary Figure 5 present the results by dividing the cohort based on those who recovered command-following during versus after rehabilitation (see online supplementary material at http://www.liebertpub.com).

\section{Patterns of functional recovery}

Multi-trajectory modeling identified four distinctive recovery patterns between Years 1 and 10, with respect to the three FIM subscales, as depicted in Figure 2. The characteristics of the four groups in the final multi-trajectory model are summarized in Table 1. Group 1 demonstrated poor recovery in all three functional domains at all time-points, with slightly greater mobility and cognitive improvement. Group $1(n=16 ; 15 \%)$ had the most severe injuries, as indicated by the latest onset of command-following and the longest acute care length of stay (LOS) among the four groups. Further, this group had the highest proportion of individuals with $\leq 12$ years of education compared with other trajectory subgroups. Group 2 showed disproportionate mobility impairment at 1 year, but robust recovery in this and the other domains over time from Years 2 to 5 and 5 to 10 . This group $(n=16 ; 15 \%)$ appeared to have the second most severe injuries in terms of time until commands were followed and may have had a high proportion of patients with diffuse axonal injury given the prevalence of motor vehicle collisions as the mechanism. A large proportion of participants in this group had an initial GCS of 3. Group 3 showed particular impairment in self-care at 1 year, but showed greater recovery in self-care and mobility than cognitive function for later follow-ups. This group $(n=20 ; 18 \%)$ had less severe injuries in terms of time to follow commands and acute care LOS. Group 4 was high functioning at 1 year for all three domains and continued to be so up until year 10 . This was the largest subgroup $(n=58 ; 53 \%)$ with more than half of the sample. While the characteristic injury for this group was very severe, this group was less severely injured than other groups with a median interval from injury to commandfollowing of 37 days and a median initial GCS of 5. Of note, there was no evidence of a pattern of deterioration up to 10 years in any functional domain or subgroup.

\section{Discussion}

In this prospective longitudinal cohort study, we monitored the trajectory of recovery over the course of 10 years in patients with severe TBI and disturbance in consciousness persisting up to the time of admission to inpatient rehabilitation. We conducted followup assessments at 1, 2, 5, and 10 years post-injury and were particularly interested in comparing outcomes in patients who regained command-following ability before 28 days post-injury and those who recovered later. We also sought to identify distinct patterns of functional recovery during the 10-year time frame. Despite an inability to follow commands at the time of inpatient rehabilitation admission, the majority of the sample achieved independence in daily functional activities across self-care, mobility, and cognitive domains. Improvement was observed at each assessment epoch, with recovery of functional independence continuing between 5 and 10 years post-injury.

We identified four distinct recovery patterns associated with varied demographic and injury severity (i.e., time to commandfollowing) characteristics: globally poor functional recovery $(15 \%)$; robust recovery of functional cognitive status and self-care ability with delayed recovery of mobility (15\%); recovery of mobility and self-care ability with residual functional cognitive limitations (18\%); and globally good functional recovery (53\%). Rate of recovery is known to be an important prognostic variable, ${ }^{19,20}$ and thus it is not surprising that those who regained commandfollowing prior to 28 days had more favorable recovery trajectories and outcomes at all time-points than those who did not. These findings extend the findings of prior longitudinal studies that report improvement across rehabilitation outcomes at 5-years postinjury. ${ }^{10,13}$ Although the sample patients from racial and ethnic minorities was small, especially when further divided into recovery pattern groups, it is notable that there were no blacks or Hispanics participants in the least recovered group (Group 1). Given the small subsample of these participants, this could simply be due to chance; however, future research should assess whether there might be bias in admitting more severely affected minority patients to rehabilitation at the outset or in survival from severe TBI during acute care. The minority patients enrolled in this study were comparable in severity to the whites, at least as defined by not following commands at rehabilitation admission.

There has been a concern about decline in function over time in the years following TBI. ${ }^{14,21}$ In a study of persons with moderate and severe TBI that was not restricted to participants without command following at the time of rehabilitation admission, Corrigan and colleague ${ }^{15}$ found $30 \%$ declined in function and $20 \%$ expired between Year 1 or 2 and the 5-year post-injury mark. The present study did not find evidence of a pattern of deterioration up to 10 years in any functional domain or subgroup. Inspection of individual level data revealed only a few isolated instances of decline. There were four instances of decline from independence in Year 5 to needing some assistance in Year 10 spread evenly across the two TFC subsamples. Areas of decline involved problem solving, memory comprehension and wheelchair mobility/walking. The cause for decline in independence for these few instances is not known, nor is it known if the decrease in independence reflects a permanent or progressive decline versus temporary change, such as might occur due to temporary illness or as a function of measurement variability. The instances of decline do not necessarily represent the same people at each epoch. For example, one individual may have declined in Year 5 and 10 while another improved over the same interval, giving the appearance of no change in 


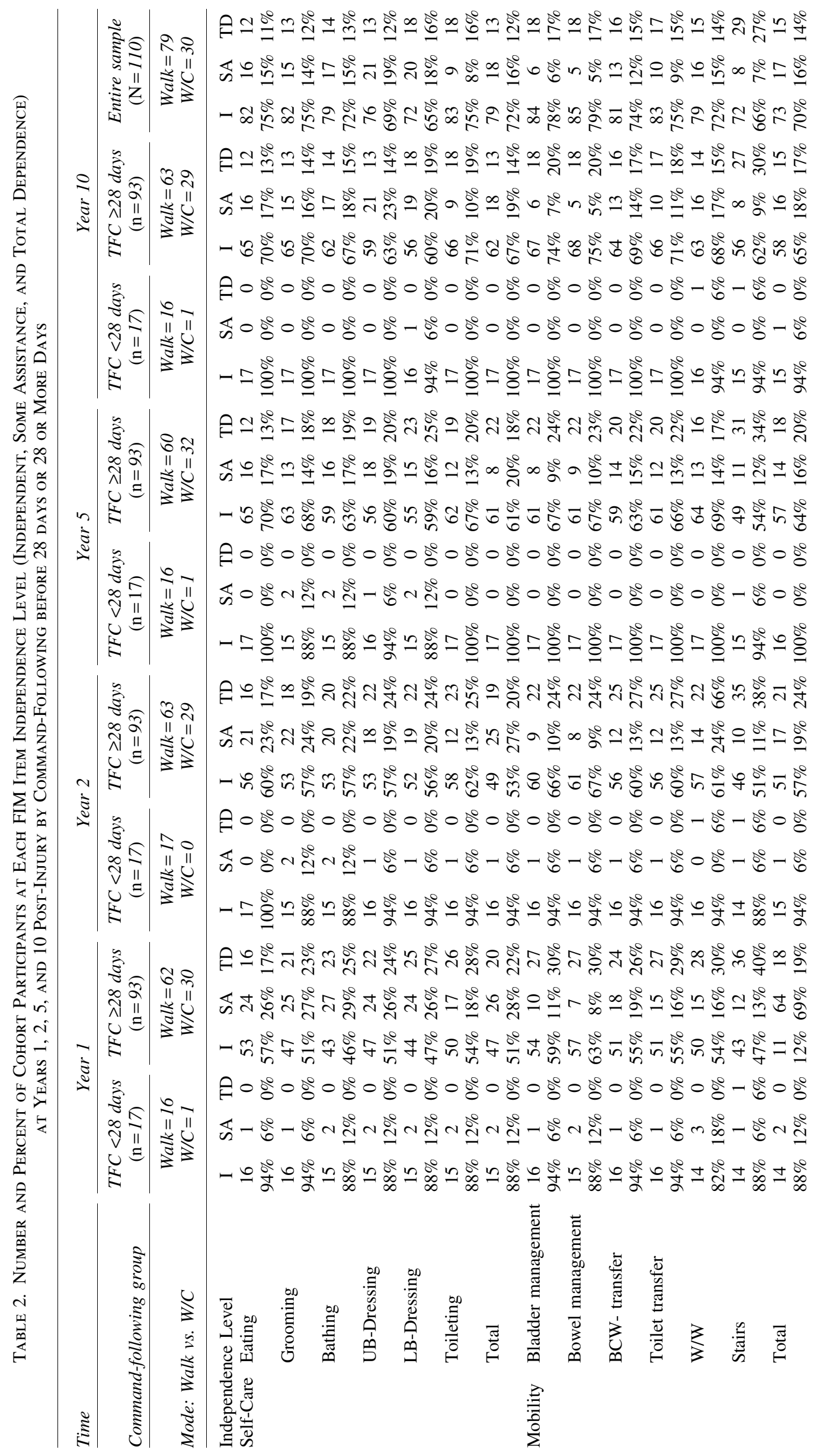




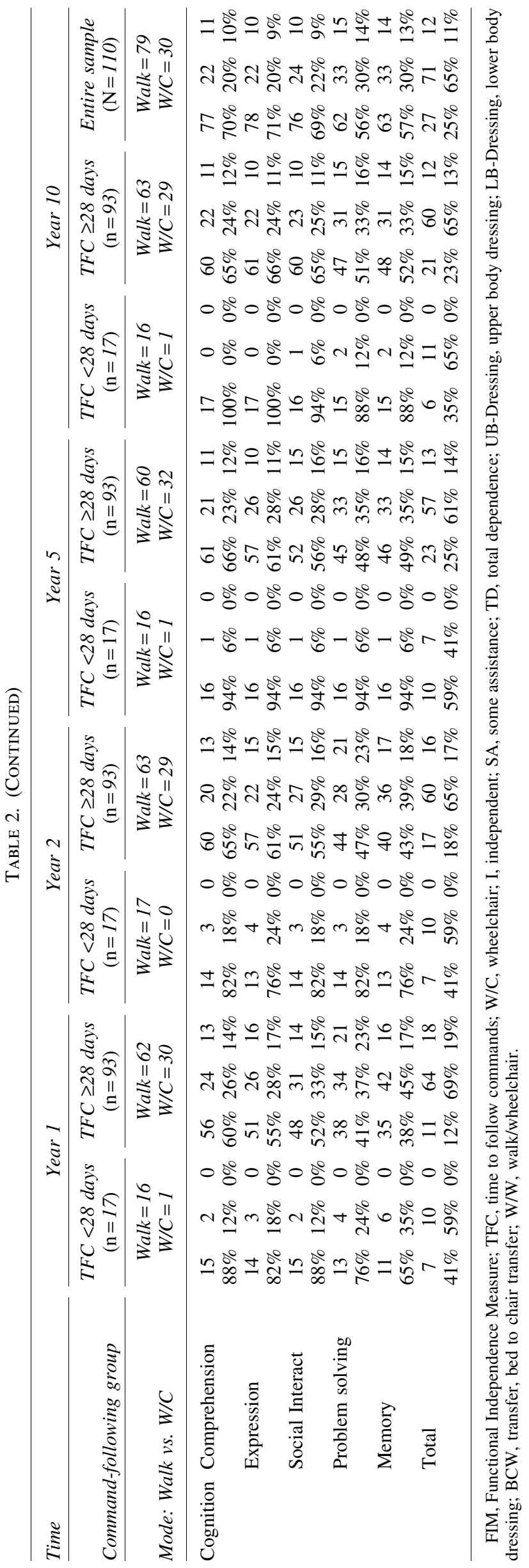



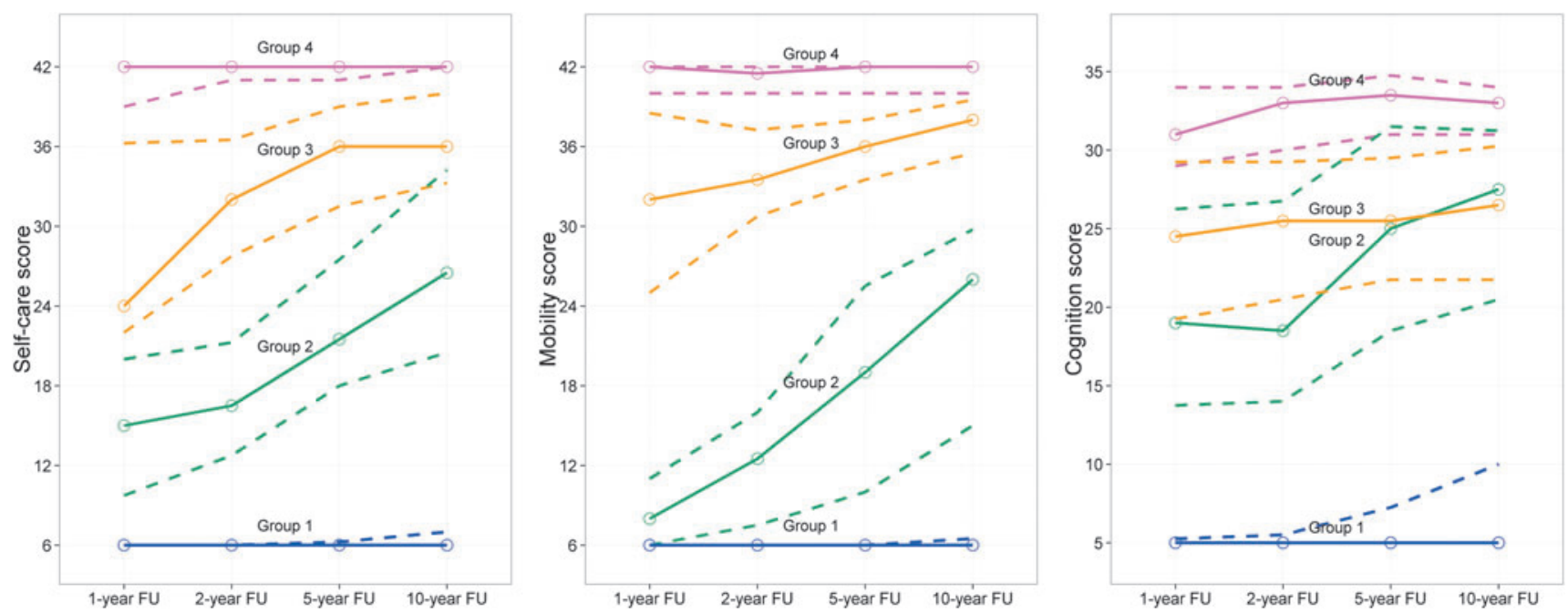

FIG. 2. Multi-trajectory modeling of recovery patterns. Modeling revealed four distinct recovery patterns. Group 1 demonstrated poor recovery in all three functional domains at all time-points, with slightly greater cognitive and mobility improvement. Group 2 showed disproportionate mobility impairment at 1 year, but robust recovery in this and the other domains over time. Group 3 showed particular impairment in self-care at 1 year, but showed greater recovery in self-care and mobility than cognitive function for later follow-ups. Group 4 was high functioning at 1 year for all three domains, which continued through Year 10.

independence for the group. It is important to note that this study did not include the 91 individuals who expired before the 10-year mark, including 14 deaths between Years 5 and 10. This current study focuses solely on function (FIM) across years, requiring functional data at each of the intervals. Those who died during the 10-year follow up period could not be included in the study as they did not have FIM data at each of the four required follow up intervals. Data on death among individuals in the TBIMS cohort who are unable to follow commands at the time of rehabilitation admission have been previously studied using the TBIMS cohort. ${ }^{22}$

Prognostic formulations developed by clinicians and payers in relation to persons with TBI of the severities included in this study sample, particularly in the early post-injury period, need to incorporate the present study findings-and to avoid the tendency towards prognostic and therapeutic nihilism that has become too common in this area of medicine in recent decades. Table 1 and Figure 2 serve as useful tools for providers when considering the potential for recovery. Supplementary Figures $1-5$ visually illustrate the proportion who are independent at each epoch using easy to understand daily activities. These figures may also aid in family education or counseling.

These data expand knowledge of long-term outcome after severe TBI and have direct implications for clinical care. Similar multicenter studies are needed to inform clinical decision-making in patients with non-traumatic brain injuries, as even less is known about long-term outcome in this population. ${ }^{23}$

\section{Study Limitations}

Several limitations should be considered in interpreting and applying the study findings. The first consideration is statistical power, particularly for the analyses of the smaller subgroups. This limited the ability to test the significance of observed FIM changes in individual items. Another consideration is the effect of possible selection bias. This study only included individuals treated at an acute care hospital and transferred directly to acute inpatient rehabilitation for specialized brain injury care. Admission to acute inpatient rehabilitation may not be offered or payment authorized for many who are not following commands. Consequently, it is unclear whether patients who are admitted to rehabilitation despite not following commands may differ from those who are not, or the degree to which intensive rehabilitation influenced the observed recovery trajectories. Further selection bias is possible through missed visits by requiring participant data across all follow up intervals, and survival bias due to exclusion of deaths. While improvement appears to continue over a decade of time among those who survive, this is counter balanced by a reasonably high mortality rate and loss to follow up raising the possibility of survivorship and ascertainment bias.

The subsample categorized as TFC 28 days or longer includes those who did command-following recover at some time-point and those who never regained command-following. As a consequence, the findings do not indicate how later recovery of commandfollowing may alter subsequent functional achievements.

Some change may be due to measurement artifact. The FIM is an observational rating scale and follow-up FIM scores were based on self or proxy report. A change in rating from independent to need for some level of assistance may reflect differences in opinion across persons interviewed from one epoch to the next, or changes in perception by the same person.

Health policies, available technologies, research evidence, practice guidelines, and clinical care practices certainly have changed over the time, both during the 10-year follow up period for each individual and during the enrollment period for this study. Such factors may have influenced the outcomes and outcome trajectories of the study participants.

Finally, there may be unknown/unmeasured treatment differences between the TFC recovery groups. In general, the later TFC recovery groups had longer acute care length of stay, and the earlier TFC recovery groups had longer rehabilitation length of stay. It is unknown if the groups differed on receipt of therapy services and specialized medical follow up in the years that followed rehabilitation discharge. It is possible that those with earlier TFC recovery were perceived to have greater rehabilitation potential and therefore received more rehabilitation services over the years postinjury. What outcomes might have been observed with different 
amounts and types of services is unknown. It is also possible that the longer rehabilitation stay permitted for those with earlier TFC recovery allowed for a better chance and/or longer time to recover command-following.

\section{Next Steps}

The findings reported here represent a complex interplay between the natural history of severe brain injury, the effects of attitudes about prognosis, and healthcare and rehabilitation access. Negative functional trajectories coupled with negative caregiver and professional attitudes may contribute to service denials or the decision to withdraw care, either of which may contribute to further functional decline or mortality. Conversely, positive functional trajectories may drive more positive attitudes and greater service access, contributing to further functional improvement. Thus, future longitudinal research with larger samples should attempt to study the interplay over time between care transitions and functional change, to isolate the role that aggressive rehabilitation care can play in shaping long-term outcome.

\section{Conclusion}

A substantial proportion of patients who are unable to follow commands upon inpatient rehabilitation admission achieve independence in cognitive, mobility, and self-care functions with improvements evident up to 10 years post-injury. A greater proportion of those who recovered command-following early (i.e., $<28$ days) achieved independence. While most functional recovery occurs by 1 -year post-injury, improvements may occur over a more prolonged interval, particularly in those who regain commandfollowing after 28 days post-injury. Late functional decline appears to be infrequent in this population that survived to Year 10. These findings suggest individuals with DOC may benefit from ongoing functional monitoring and updated care plans during the post-acute phase. Our findings can inform clinical decision-making, prognosis, and treatment planning.

\section{Acknowledgments}

The views, opinions, and/or findings contained in this article are those of the authors and should not be construed as an official Department of Defense position or any other federal agency, policy, or decision unless so designated by other official documentation. The contents of this publication do not necessarily represent the policy of NIDILRR, ACL, HHS, Veterans Affairs, and you should not assume endorsement by the Federal Government.

The contents of this publication were developed under grants from the National Institute on Disability, Independent Living, and Rehabilitation Research (NIDILRR; grant numbers, DPTBI17000014; 90DP0036 and 90DRTB0002; 90DPTB0011, 90DP0039), which is a Center within the Administration for Community Living (ACL), Department of Health and Human Services (HHS).

The Polytrauma Rehabilitation Center Traumatic Brain Injury (TBI) Model System collaboration is funded through an Interagency Agreement between the Department of Veterans Affairs and the Department of Health and Human Services (National Institute on Disability, Independent Living, and Rehabilitation Research). This research is sponsored by VHA Central Office VA TBI Model System Program of Research, and Subcontract from General Dynamics Health Solutions (W91YTZ-13-C-0015) from the Defense and Veterans Brain Injury Center within the Defense Health Agency, and U.S. Department of Veterans Affairs Health Services
Research and Development COIN grant (1 I50 HX001233-01; CINDRR).

The authors acknowledge and appreciate assistance from Marie Elise Saylors, MPH.

Author contributions: Flora M. Hammond, MD—study concept, design and oversight, acquisition and interpretation of data, drafting/revising the manuscript, critical revision of the manuscript for important intellectual content; Risa Nakase-Richardson, PhDstudy design, acquisition and interpretation of data, drafting/revising the manuscript, critical revision of the manuscript for important intellectual content; Joseph T. Giacino, PhD—study design, acquisition and interpretation of data, drafting/revising the manuscript, critical revision of the manuscript for important intellectual content; Mark Sherer, $\mathrm{PhD}$ - study design, acquisition and interpretation of data, drafting/revising the manuscript, critical revision of the manuscript for important intellectual content; Ross Zafonte, DO-study design, analysis and interpretation of data, drafting/ revising the manuscript, critical revision of the manuscript for important intellectual content; John Whyte, MD, PhD—study design, analysis and interpretation of data, drafting/revising the manuscript, critical revision of the manuscript for important intellectual content; David Arciniegas, MD—study design, analysis and interpretation of data, drafting/revising the manuscript, critical revision of the manuscript for important intellectual content; Xinyu Tang, PhD—study design, analysis and interpretation of data, drafting/revising the manuscript, critical revision of the manuscript for important intellectual content.

\section{Author Disclosure Statement}

The data utilized in this manuscript and the authors' contributions were made possible by grant support from the National Institute on Disability, Independent Living and Rehabilitation Research (NIDILRR), Administration for Community Living. The contents of this manuscript do not necessarily represent the policy of the U.S. Department of Health and Human Services, and endorsement by the federal government should not be assumed.

Dr. Hammond has consulted within the past 12 months for Avanir Pharmaceuticals, Inc. as a member of the PRISM II Steering Committee; has stock ownership in healthcare companies including Abbvie Inc SHS, Eli Lilly and Co, GlaxoSmithKline PLC ADR, Exchange Traded Funds, and Mutual Funds; receives royalties from Lash Publishing and Demos Medical; and receives research support from the National Institute on Disability Independent Living and Rehabilitation Research. She serves on the editorial board of the Journal of Head Trauma Rehabilitation.

Dr. Nakase-Richardson is an Associate Professor of in the Morsani College of Medicine at the University of South Florida and employed as a Clinician Investigator within the Department of Veterans Affairs. She is funded by General Dynamics Health Solutions (W91YTZ-13-C-0015) from the Defense and Veterans Brain Injury Center, Defense Health Agency, the Patient Centered Outcomes Research Institute (PCORI R-1511-33005), Department of Veterans Affairs Rehabilitation Research and Development (VA RR\&D; 1 I21 RX001923-01A1), Health Services Research and Development (VA HSRD; 1 I50 HX001233-01), and Clinical Sciences Research and Development (W81XWH-13-2-0095). In the past year, she has received honoraria or travel compensation for presentations or grant review activities from the University of Pittsburgh, Western Michigan Brain Injury Network, Craig Hospital, Center for Neuroscience and Regenerative Medicine and Congressionally Directed Medical Research Programs. 
Dr. Giacino is a member of the American Congress of Rehabilitation Medicine, the Brain Injury Special Interest Group, and the Disorders of Consciousness Task Force; serves on a scientific advisory board for TBI Model Systems National Data and Statistical Center; has received funding for travel from the U.S. Department of Defense for a meeting related to the TBI Endpoint Development Project, the National Institute on Neurological Disorders and Stroke for the Traumatic Brain Injury Model Systems Project Directors meeting, and for a meeting related to the Transforming Research and Clinical Knowledge in Traumatic Brain Injury study, the National Institute on Disability, Independent Living and Rehabilitation Research, the American Academy of Physical Medicine and Rehabilitation, the One Mind Foundation, the James S. McDonnell Foundation for a meeting related to the Recovery of Consciousness After Severe Brain Injury study, the Barbara Epstein Foundation, and the International Brain Injury Association; has received a cash donation from the Epstein Foundation for a hospital clinical program that he directs and for serving on a team that provided clinical consultation services to an overseas patient who sustained severe brain injury; has served as an editor for the Journal of Head Trauma Rehabilitation; has received honoraria from the One Mind Foundation, Holy Cross Hospital (Surrey, UK); HealthSouth Braintree Hospital, Western Michigan Brain Injury Network, George Washington University Medical School, Association of Academic Physiatrists, Mayo Clinic, Kennedy-Krieger Institute, and Magill's Medical Guide; performs clinical procedures as $10 \%$ of his clinical effort in his role as Director of Spaulding Rehabilitation Network Disorders of Consciousness Program and neuroimaging as a principal investigator on two neuroimaging studies for $30 \%$ of his research effort; received financial support from the NIH-National Institute on Neurological Disorders and Stroke (NINDS) for Central Thalamic Stimulation for Traumatic Brain Injury, U.S. Department of Defense for TBI Endpoint Development Project, the Huperzine A for the Treatment of Cognitive, Mood and Functional Deficits After Moderate and Severe TBI study, the INjury and TRaumatic STress (INTRuST) Consortium Neuroimaging Acquisition and Archival study, the National Institute on Disability, Independent Living and Rehabilitation Research (NIDILRR) for the Spaulding HarvardTraumatic Brain Injury Model System and for Multicenter Evaluation of Memory Remediation after traumatic Brain Injury with Donepezil, National Institute for Neurologic Disorders and Stroke (NINDS) for Transforming Research and Clinical Knowledge in Traumatic Brain Injury study, James S. McDonnell Foundation for Study of Recovery of Consciousness After Severe Brain Injury, Barbara Epstein Foundation, and the Spaulding Rehabilitation Hospital Department of Physical Medicine and Rehabilitation; and has acted as a witness with regard to a legal proceeding.

Dr. Sherer is Associate Vice President for TIRR Memorial Hermann. His research is funded by NIDILRR and NIH. He serves on the editorial boards of Journal of Head Trauma Rehabilitation and Rehabilitation Psychology. In the past year, he has received honoraria from the Council on Brain Injury, Center of Innovation on Disability and Rehabilitation Research, and the International Neuropsychological Society, and has been reimbursed for travel expenses by the Council on Brain Injury, the North American Brain Injury Society, Center of Innovation on Disability and Rehabilitation Research, and the International Neuropsychological Society. He serves on the Advisory Board for the Center of Innovation on Disability and Rehabilitation Research at Malcolm Randall VA Medical Center.

Dr. Zafonte received research support from NIDILRR, NIH and USARMC: NIDILRR: 90DP0039-03-00, 90SI5007-02-04,90 D P0060; USAMRC-W81XWH-112-0210,NIH: 4 U01NS086090-04;
5R24HD082302-02;5U01NS091951-03. He also serves as coprincipal investigator on a $\mathrm{T}-32$ and receives funding from the Football Players Health Study at Harvard University, which is funded by the NFL Players Association. Dr Zafonte received royalties from: 1) Oakstone for an educational CD- Physical Medicine and Rehabilitation a Comprehensive Review; and 2) Demos publishing for serving as coeditor of the text Brain Injury Medicine. Dr Zafonte serves on the Scientific Advisory Board of Myomo, Oxeia Biopharma, and EIMINDA. He also evaluates patients in the MGH Brain and BodyTRUST Program, which is funded by the NFL Players Association.

Dr. Whyte directs the Moss Rehabilitation Research Institute. He receives funding from the National Institute on Disability, Independent Living, and Rehabilitation Research, the National Institutes of Health, and the Patient Centered Outcomes Research Institute. He serves as President of the Foundation for Physical Medicine and Rehabilitation, and on the Board of Trustees for the Association of Academic Physiatrists. He is a member of the Editorial Boards of the Journal of Head Trauma Rehabilitation and the American Journal of Physical Medicine and Rehabilitation. He receives honoraria and travel support from multiple not-for-profit institutions for speaking engagements and provides expert medical legal testimony on occasional cases of patients with disorders of consciousness.

Dr. Arciniegas receives research support from the National Institute on Disability, Independent Living, and Rehabilitation Research (H133A120020, H133A130047), Department of Veterans Affairs (CX000239), and Patient-Centered Outcomes Research Institute-Eugene Washington PCORI Engagement Award (EAIN7136), and the Marcus Institute for Brain Health at the University of Colorado School of Medicine; serves on the scientific advisory board for the Traumatic Brain Injury Model Systems National Data and Statistical Center; is the Editor of the Journal of Neuropsychiatry and Clinical Neurosciences and in this and related roles receives support from American Psychiatric Association Publishing, Inc.; serves (uncompensated) on the editorial boards of Journal of Head Trauma Rehabilitation and Brain Injury; receives royalties from Cambridge University Press and Demos Medical Publishing; and in the past 12 months, has received honoraria and travel expense reimbursements from Indiana University School of Medicine, Weill Cornell Medicine of Cornell University, and employment income from the Center for Mental Health.

Dr. Tang is an Associate Professor of Biostatistics Program in the Department of Pediatrics at the University of Arkansas for Medical Sciences. She is funded by General Dynamics Health Solutions (W91YTZ-13-C-0015) from the Defense and Veterans Brain Injury Center, Defense Health Agency, and has a contract with Craig Hospital for providing biostatistical support for analytic projects under Craig's VA contract VA119A-16-C-0083, Traumatic Brain Injury (TBI) Registry and Rehabilitation Database. In the past year, she has received honoraria from serving as grant reviewer for NIH and HRSA.

\section{References}

1. Hammond, F., Natarajan, S., Toomer, A., Huynh, T., and Norton, J. (2009). Accuracy of predicting 6-month outcome at 48 hours following severe traumatic brain injury: a pilot study. J. Neurotrauma 26, A1-101.

2. Izzy, S., Compton, R., Carandang R., Hall W., and Muehlschlegel S. (2013). Self-fulfilling prophecies. NeuroCrit Care 19, 347-363.

3. Fins, J.J. (2012). Severe brain injury and organ solicitation: a call for temperance. Virtual Mentor 14, 221-226.

4. Kotwica, Z. and Jakubowski, J.K. (1995). Head-injured adult patients with GCS of 3 on admission: who have a chance to survive? Acta Neurochir (Wien) 133, 56-59. 
5. Levy, M.L., Masri, L.S., Lavine, S., and Apuzzo, M.L. (1994). Outcome prediction after penetrating craniocerebral injury in a civilian population: aggressive surgical management in patients with admission Glasgow Coma Scale scores of 3, 4, or 5. Neurosurgery 35, 7785 .

6. Giacino, J.T. and Kalmar, K. (1997). The vegetative and minimally conscious states: a comparison of clinical features and functional outcome. J. Head Trauma Rehabil. 12, 36-51.

7. Katz, D.I., Polyak M., Coughlan, D., Nichols, M., and Roche, A. (2009) Natural history of recovery from brain injury after prolonged disorders of consciousness: outcome of patients admitted to inpatient rehabilitation with 1-4 year follow up. Prog. Brain Res. 177, 73-88.

8. Giacino, J., Ashwal, S., Childs, N., Cranford, R., Jennett, B., Katz, D.I., Kelly, J.P., Rosenberg, J.H., Whyte, J., Zafonte, R.D., and Zasler, N.D. (2002). The minimally conscious state: definition and diagnostic criteria. Neurology 58, 349-353.

9. Luaute, J., Maucort-Boulch, D., Tell, L., Quelard, F., Sarraf, T., Iwas, J., Boisson, D., and Fischer, C. (2010) Long-term outcomes of chronic minimally conscious and vegetative states. Neurology 75, 246-252.

10. Nakase-Richardson, R., Whyte, J., Giacino, J.T., Pavawalla, S., Barnett, S.T., Yablon, S.A., Sherer, M., Kalmar, K., Hammond, F., Greenwald, B., Horn, L.J., Seel, R.T., McCarthy, M., Tran, J., and Walker, W. (2012). Longitudinal outcome of patients with disordered consciousness in the NIDRR TBI Model Systems Programs. J. Neurotrauma $29,59-65$.

11. Lammi, M.H., Smith, V.H., Tate, R.L., and Taylor, C.M. (2005). The minimally conscious state and recovery potential: a follow-up study 2 to 5 years after traumatic brain injury. Arch. Phys. Med. Rehabil. 86, 746-754.

12. DeGuise, E., LeBlanc, J., Feyz, M., Meyer, K., Duplantie, J., Thomas, H., Abouassaly, M., Champoux, M.C., Couturier, C., Lin, H., Lu, L. Robinson, C., and Roger, E. (2008). Long-term outcome after severe traumatic brain injury: the McGill Interdisciplinary Prospective Study. J. Head Trauma Rehabil. 23, 294-303.

13. Whyte, J., Nakase-Richardson, R., Hammond, F.M., McNamee, S., Giacino, J.T., Kalmar, K., Greenwald, B.D., Yablon, S.A., and Horn, L.J. (2013). Functional outcomes in traumatic disorders of consciousness: 5-year outcomes from the National Institute on Disability and Rehabilitation Research Traumatic Brain Injury Model Systems. Arch. Phys. Med. Rehabil. 94, 1855-1860.

14. Corrigan, J.D. and Hammond, F.M. (2013). Traumatic brain injury as a chronic health condition. Arch. Phys. Med. Rehabil. 94, 1199-1201.

15. Corrigan, J.D., Cuthbert, J.P., Harrison-Felix, C., Whiteneck, G.G. Bell, J.M., Miller, A.C., Coronado, V.G., and Pretz, C.R. (2014). US population estimates of health and social outcomes 5 years after re- habilitation for traumatic brain injury. J. Head Trauma Rehabil. 29, E1-E9.

16. Hamilton, B.B., Granger, C.V., Sherwin, F.S., Zielezny, M., and Tashman, J.S. (1987). A uniform national data system for medical rehabilitation, in: Rehabilitation Outcomes: Analysis and Measurement, MJ Fuhrer (ed). Baltimore: Brooks: Baltimore, MD, pps. 137147.

17. Bogner, J.A., Whiteneck, G.G., MacDonald, J., Juengst, S.B., Brown, A.W., Phillippus, A.M., Marwitz, J.H., Lengenfelder, J., Mellick, D., Arenth, P., and Corrigan, J.D. (2017). Test-retest reliability of traumatic brain injury outcome measures: a traumatic brain injury model systems study. J. Head Trauma Rehabil 32, E1-E16.

18. Nagin, D.S., Jones, B.L., Passos, V.L., and Tremblay RE. (2016) Group-based multi-trajectory modeling. Stat. Methods Med. Res. 27, 2015-2202.

19. Giacino, J., Kezmarsky, M., DeLuca, J., and Cicerone, K. (1991). Monitoring rate of recovery to predict outcome in minimally responsive patients. Arch. Phys. Med. Rehabil. 72, 897-901.

20. Whyte, J., Katz, D., Long, D., DiPasquale, M., Polansky, M., Kalmar, K., Giacino, J., Childs, N., Mercer, W., Novak, P., Maurer, P., and Eifert, B. (2005). Predictors of outcome in prolonged posttraumatic disorders of consciousness and assessment of medication effects: a multicenter study. Arch. Phys. Med. Rehabil. 86, 453-462.

21. Institute of Medicine. (2009). Gulf War and Health: Volume 7. Longterm Consequences of Traumatic Brain Injury. The National Academies Press: Washington, DC.

22. Greenwald, B.D., Hammond, F.M., Harrison-Felix, C., NakaseRichardson, R., Howe, L.L.S., and Kreider, S. (2015). Mortality following traumatic brain injury among individuals unable to follow commands at the time of rehabilitation admission: a NIDRR TBI model systems study. J. Neurotrauma 32.

23. Estraneo, A., Moretta, P., Loreto, V., Lanzillo, B., Santoro, L., and Trojano, L. (2010) Late recovery after traumatic, anoxic, or hemorrhagic long-lasting vegetative state. Neurology 75, 239-245.

Address correspondence to:

Flora M. Hammond, MD

Department of Physical Medicine and Rehabilitation Indiana University School of Medicine

4141 Shore Drive Indianapolis, IN 46254

E-mail: flora.hammond@rhin.com 